\title{
Stroke and Constipation
}

\section{-Coincidence or Interrelated?}

\author{
Jayaprada Kasaraneni, Margaret Hayes
}

Department of Family Medicine, Oregon Health \& Science University, Portland, OR, USA

Email: kasaranenij@gmail.com

Received 18 September 2014; revised 3 November 2014; accepted 18 November 2014

Copyright (C) 2014 by authors and Scientific Research Publishing Inc.

This work is licensed under the Creative Commons Attribution International License (CC BY).

http://creativecommons.org/licenses/by/4.0/

(c) (i) Open Access

\begin{abstract}
Emile Gautier once said, "Freedom of the bowels is the most precious, perhaps even the most essential, of all freedoms-one without which little can be accomplished." This paper will explore the neuronal physiology, pathophysiology, theories regarding the correlation between stroke and constipation along with a few treatment options. Patients often recovering from stroke complain of constipation and it is most likely attributed to changes in diet, ambulation, or fluid balance. However, there are not many studies to reflect the correlation between other less significant symptoms and stroke presentation.
\end{abstract}

\section{Keywords}

Stroke, Constipation, Treatment of Constipation, Studies on Stroke

\section{Introduction to Constipation}

Constipation is defined as having less than 3 bowel movements per week, difficulty with defecation, or a sense of incomplete evacuation. The two criteria most often used to diagnose constipation are the Bristol Stool Chart and the Rome III Criteria. The Bristol Stool Chart is a visual guide for stool classified by appearance utilizing a graphic chart of seven variables. More widely used, Rome III Criteria [1] are used for clinical diagnosis and clinical trials.

1. The Criteria must include two or more of the following:

a. Straining during at least $25 \%$ of defecations

b. Lumpy or hard stools in at least $25 \%$ of defecations

c. Sensation of incomplete evacuation for at least $25 \%$ of defecations

d. Sensation of anorectal obstruction/blockage for at least $25 \%$ of defecations

e. Manual maneuvers to facilitate at least $25 \%$ of defecations (e.g., digital evacuation, support of the pelvic floor) 
f. Fewer than three defecations per week

2. Loose stools are rarely present without the use of laxatives.

3. Insufficient criteria for irritable bowel syndrome

\section{Neuronal Physiology of the Gastrointestinal Tract}

The gastrointestinal tract, containing 200 to 500 million neurons [2] is nicknamed the name "Second Brain". The gastrointestinal neuronal physiology consists of both intrinsic and extrinsic innervations. The enteric nervous system including myenteric, submucosal and mucosal innervations makes up the intrinsic pathways. Myenteric neurons control GI motility, while submucosal neurons control GI blood flow and intestinal ion transport. The extrinsic innervations consist of parasympathetic and sympathetic neurons. The parasympathetic fibers, originating from the vagus nerve, course along branches of the superior mesenteric artery to reach the small intestine and proximal colon through the splenic flexure. Parasympathetic fibers from the sacral plexus [3] innervate the distal colon.

\section{Introduction to Stroke}

Cerebrovascular accident [4] or stroke is a neurological deficit of abrupt onset attributable to a focal vascular cause, either embolic or hemorrhagic. The clinical suspicion of stroke is confirmed by imaging, typically computed tomography (CT) scans, to confirm the location, extent and type of vascular accident. Neurologic manifestations typically occur within a few minutes of ischemia onset due to loss of available glycogen for adequate cellular metabolism. The return of blood flow within 24 hours is associated with reversal of the neurologic deficit, whereas failure to re-establish blood flow within 24 hours is associated with permanent deficit.

Stroke Classification:

1. Ischemic Stroke is caused by occlusion of an intracranial vessel, typically from embolic tissue originating from the left ventricle of the heart or carotid arteries, that causes decreased blood flow to the region it supplies leading to necrotic and/or apoptotic cell death in the brain.

a. A decrease in blood flow to zero results in cellular death within 4 - 10 min.

b. Values of $<16-18 \mathrm{~mL}$ blood flow/100 $\mathrm{g}$ of brain tissue causes infarction within the hour

c. Values $<20 \mathrm{~mL}$ blood flow/100 g of brain tissue causes ischemia without infarction in several hours.

Common types of Ischemic Stroke include:

- Cardio embolic Stroke

- Artery to Artery embolic stroke

- Small vessel Stroke

2. Hemorrhagic Stroke: Bleeding in intracerebral hemorrhage is usually derived from arterioles or small arteries. The bleeding is directly into the brain, forming a localized hematoma that spreads along white matter pathways. Accumulation of blood occurs over minutes or hours; the hematoma gradually enlarges by adding blood at its periphery like a snowball rolling downhill.

The most common causes of ICH are:

- Hypertension, trauma, bleeding diatheses, amyloid angiopathy, illicit drug use (mostly amphetamines and cocaine), and vascular malformations

Less frequent causes include:

- Bleeding into tumors, aneurysmal rupture, and vasculitis

3. Transient Ischemic Attacks: The term transient ischemic attack (TIA) was first introduced in the early 1950s based upon the recognition that transient focal loss of neurologic function often preceded strokes. The classic definition of a TIA is the sudden onset of a focal neurologic symptom and/or signs that

d. last less than 24 hours

e. is presumably brought on by a transient decrease in blood supply, which renders the brain ischemic in the area producing the symptom

f. restoration of blood flow is timely enough to make the ischemia transient and avoid infarction.

\section{Neuronal Physiology Involved in Stroke}

The neuron, the basic functional unit of the nervous system, is responsible for the transmission of signals from the periphery to the brain (afferent neurons) and responses from the brain to the periphery (efferent neurons). An 
occlusion with decreased blood flow leads to inhibition of metabolic activity and the availability of neurotransmitters to the affected area including glutamate, GABA acetylcholine, dopamine, norepinephrine, and serotonin.

As glutamate and aspartate increase in the brain, there is over-excitation of neurotransmission leading to hypoxic-ischemic neuronal injury. This process is known as "excitotoxicity" [5]. It is due to a decrease in cell energy stores due to over excitation of neurons. The increase in glutamate in extracellular space in decreased energy states opens the calcium channels associated with NMDA and AMPA receptors. Intracellular calcium causes activation of destructive enzymes such as proteases, lipases, and endonucleases causing the release of cytokines resulting in cell damage.

Inflammatory response leads to leukocyte recruitment to the ischemic areas and occurs within 30 minutes of ischemia to any area [6]. Leukocytes in the area have two affects. First producing a mechanical obstruction followed by activation of vasoactive substances including oxygen free radicals, arachidonic acid metabolites and nitric acid. This leads to increased vasodilation (state where since the next item is vasoconstriction), peripheral vasoconstriction, vascular permeability, platelet aggregation, leukocyte adherence to endothelial wall and immunoregulation. Endothelial cells also modulate the actions of vasoactive agents, which influence the vascular tone of microcirculation [6].

\section{Constipation: Numbers}

\section{General Population:}

Prevalence-Approximately 15\%-more common in females and elderly [3]. In a study investigating 15,000 women, $14 \%-27 \%$ were found to be constipated, most were elderly [3]. Walking less than $0.5 \mathrm{~km}$ a day increases the risk of constipation in the elderly [3]. Thirty-five grams of dietary fiber is associated with normal stooling [3].

\section{Stroke Population:}

Prevalence of Constipation after Stroke is 30\% to 60\% [7].

In a study from China [8] $(\mathrm{n}=723)$ post stroke constipation was noted in $34.6 \%$.

a) $31 \%$ of ischemic stroke patients had constipation.

b) $44.8 \%$ of hemorrhagic stroke patients had constipation.

c) $47.4 \%$ of cerebral hemorrhage and infarction patients had constipation.

In the same study [8], patients were divided based on the stages after stroke that presented with constipation were:

a) $41.6 \%$ had constipation in acute phase.

b) $31.5 \%$ had constipation in recovery phase.

c) $22.6 \%$ had constipation in sequelae phase.

In a separate study from China [9], new onset constipation was $55.2 \%$ at 4 weeks post stroke. This incidence was due to dependence and use of bedpan for defecation. A study from late 1970's to early 1980's found that GI complications following stroke occurred in about $24 \%$ of cases. Of these, $63.5 \%$ had a hemorrhage into the lumen of the alimentary tract, most often within the first week [10]. Incidence of constipation was significantly higher in patients with basal ganglia involvement than in other sites [10]. New onset constipation is seen in 55\% of patients within a month after first stroke [11]. The development of constipation predicts a poor outcome at 12 weeks in patients with moderately severe stroke [9]. New onset constipation occurs in $30 \%$ of hemiplegic patients and has no relationship with the site or severity of stroke [7].

\section{Theories of Correlation}

- Elderly Patients: Stroke patients more often than not are in the older age group. Atherosclerosis makes this population more prone to strokes. These patients are often on multiple medications and may be dehydrated and immobile on admission to the hospital. As people age physiologic changes occur throughout the body. The changes in the gastrointestinal system include decreased myenteric function and inhibitory nerve input to colon and increased collagen deposition in the left colon, and binding of plasma endorphins to intestinal receptors. A decrease in myenteric function leads to constipation, due to decreased motility of the GI tract. Collagen deposits in the colon cause a decrease in circulation to the bowel, leading to a decrease in peristaltic movements. These two factors, along with new onset stroke, can cause an even further decrease in GI motility due to stress on the body. Physical inactivity, however, does not have a correlation between being consti- 
pated and stroke [11]. Physical activity has been shown to have positive effects on the body including a decrease in incidence of stroke. The role of rehabilitation is proven in post stroke therapy. The role of exercise in resolving constipation however is not significant. Therefore, the role of physical activity in relation to constipation and stroke is not clear.

- A number of drugs commonly used in adults are also known to cause constipation. Drugs that have constipation as a significant side effect include diuretics, iron, antihypertensives, opioids and anticholinergic agents. Medication induced constipation is most likely from a change in electrolyte and fluid balance in both the small intestine and large intestine or by a decrease in propulsion due to serotonergic activity. Dehydration due to a decrease in oral consumption and impaired thirst mechanisms cause a decrease in defecation urge, as there is a lesser volume in the intestine due to reabsorption of water.

- Location in the cortex: The cerebral cortex is a map of complex functions-both motor and sensory. The cortex controls most of what we take for granted such as breathing, vision, sense of judgment and all intellectual functions among others. Cerebrovascular accidents, can affect any part or lobe of the brain and such an event would present with a deficiency in the area involved. If it occurs as a small stroke or TIA, the decline would be slow and unnoticeable [12]. In cases of moderate to severe strokes, constipation cannot be the only symptom due to the cortical representation; it usually precedes or succeeds a stroke pointing towards the area of involvement most often with hemiplegia. It was hypothesized as early as 1993 [13] that cortical control of the defecation center may be located bilaterally, with a center on one side being more dominant. Cortical mapping done by transcranial magnetic stimulation has shown that centers are most likely located in both hemispheres. Therefore, it is hypothesized that if damage occurs in stroke to the dominant area, the other side may not be able to maintain the defecatory center function, causing constipation.

- Prescription drug usage: In acute post stroke stages patients are treated with multiple medications. An osmotic diuretic such as mannitol is usually the first drug employed to decrease the intracranial pressure that rises soon after a cerebrovascular accident. Mannitol typically takes effect within a few minutes and is effective in decreasing the intracranial pressure (ICP). Diuretics such as furosemide are given to prolong the effect of mannitol. This therapy requires close monitoring of fluid input and output to avoid dehydration and further risk of constipation due to excessive diuresis. Morphine is another common post-stroke medication that is widely known to cause constipation by seizing or decreasing the peristaltic movements of the intestines. This is seen more often in chronic narcotic medication users, although constipation can occur within 2 weeks of usage.

- Intestinal microbial metabolism: A recent study measured plasma and urinary levels of TMAO (trimethylamine-N-oxide), plasma choline and betaine levels in otherwise normal people before and after the use of oral broad-spectrum antibiotics for their effect on intestinal microbia. The levels of TMAO were significantly lower when compared to before use of antibiotics. Increased levels of TMAO were associated with increased risk for cardiovascular events due to atherosclerosis. TMAO is produced from phosphatidylcholine, present in eggs, liver, beef, and pork, and is metabolized by the intestinal flora. Therefore, if there is a decrease in the volume of bacterial intestinal flora, there is more TMAO circulating and leading to an increase in atherosclerosis. L-Carnithine, is an amino acid that is widely popular for use in chronic fatigue syndrome and aids in weight loss. L-Carnithine consists of trimethylamine which is further metabolized by the intestinal microbial to TMAO and increases the risk of atherosclerosis. In a study it was shown that, omnivorous human subjects produced more TMAO than did vegans or vegetarians following ingestion of L-carnithine through a microbiota-dependent mechanism. [Nature magazine]. This shows that people who consume more red meat are prone to vascular effects due to atherosclerosis.

- Dehydration: Dehydration as an independent cause of constipation following any cerebrovascular accident is prevalent, as many patients are not put on aggressive hydration due to a need to decrease the ICP and maintain diruesis. Patients with post-stroke swallowing issues have additional challenges maintaining adequate hydration. This causes a decrease in water availability for reabsorption from kidneys and intense intestinal resorption of water, causing constipation. The chloride channels in the CFTR are widely open causing water reabsorption from the intestines. This leads to a decrease in the volume present in the intestines and a decrease in stool water content and in defecation urge as discussed earlier, leading to constipation.

\section{Diagnosis}

A diagnosis of constipation is usually made by the absence of bowel movement or hard bowel movements in the 
two weeks following stroke but can present as late as four weeks. A careful history along with all medications and recent diagnosis of the patient's illness is important to determine the etiology of constipation. Some tests that can be performed are a measurement of whole gut transit time, swallowing a radio opaque contrast, and anorectal testing to check for function or pelvic floor coordination difficulty.

\section{Treatment Options}

- Fiber: High fiber food such as fruits or vegetables can be added to the diet to help increase the volume present and cause bowel movement by distension. If fiber foods cannot be consumed, fiber supplements such as bran, psyllium, or methylcellulose can be added to help increase the fiber consumed by the patient.

- Water: The Institute of Medicine determined that an adequate intake (AI) for men is roughly 3 liters (about 13 cups) of total beverages a day. The AI for women is 2.2 liters (about 9 cups) of total beverages a day.

- Pharmacologic medications [14] [4]:

a. Osmotic Laxative: The preferred option. Osmotic laxatives work by causing a water shift into the intestine. Useful when the small and large intestines are not able to maintain the osmotic gradient necessary to support normal bowel function. These may take a few days to be effective and have the potential to cause electrolyte disturbances requiring close monitoring. Osmotic laxatives include magnesium citrate, magnesium hydroxide and magnesium sulfate.

b. Lactulose: Lactulose is a sugar that is not digested. The bacteria present in the small and large intestine metabolize lactulose to form lactic, acetic, and formic acids. This acidification of the intestinal lumen and leads to water secretion into the intestine with colon distension and bowel movements.

c. Stimulant laxatives: These include docusate sodium, diphenylmethane derivatives like bisacodyl, and anthroquinones such as senna and cascara. Anthroquinones act by causing contractions of intestinal smooth muscle and increasing the bowel motility. They also cause an increase in secretion of water into the intestine. Diphenylmethane derivatives act by inhibition of water reabsorption from the intestine and causing minor peristaltic movements.

d. Castor oil: Castor oil helps relieve constipation by four different actions. These include increasing cAMP levels, increasing mucous permeability, causing Nitric oxide synthesis, and Na-K-ATPase inhibition. It is often used in preparing the bowel prior to surgical procedures.

\section{Conclusion}

Therefore, in summary, it is extremely crucial to keep vigilance on patients with stroke for a month following the event. There are several studies indicating a strong correlation as mentioned between acquiring a stroke and constipation. However, it is imperative that we conduct more retrospective studies by qualitative analysis of symptomatic presentations and the extent of stroke along with duration of presentation. Constipation should be promptly treated by one of the options mentioned to improve patient comfort. It is essential to get the patient to physical rehabilitation to enhance recovery post stroke.

\section{References}

[1] Arce, D.A., Ermocilla, C.A. and Costa, H. (2002) Evaluation of Constipation. American Family Physician, 65, 22832291.

[2] Furness, J.B. (2007) Enteric Nervous System. Scholarpedia, 2, 4064. http://dx.doi.org/10.4249/scholarpedia.4064

[3] Winge, K., Rasmussen, D. and Werdelin, L.M. (2003) Constipation in Neurological Diseases. Journal of Neurology, Neurosurgery \& Psychiatry, 74, 13-19. http://dx.doi.org/10.1136/jnnp.74.1.13

[4] Smith, W.S., English, J.D. and Johnson, S.C. (2012) Harrison’s Principles of Internal Medicine. McGraw-Hill, New York.

[5] Shah, N.D., Locke, G.R., Meek, P.D., et al. (2006) Ambulatory Care for Constipation in the United States, 1997-2004. The American Journal of Gastroenterology, 101, S220.

[6] Shah, S. Pathophysiology of Stroke. www.uic.edu/com/ferne/pdf/pathophys0501.pdf

[7] Luca, G., Domenico, P., Caterina, P. and De Sarro, G. (2012) Constipation Treatment in Neurological Disorders, Constipation-Causes, Diagnosis and Treatment. Anthony Catto-Smith (Ed.), InTech. http://www.intechopen.com/books/constipation-causes-diagnosis-and-treatment/constipation-treatment-in-neurological -disorders 
[8] Cai, W.Z., Wang, L., Guo, L., Wang, J.X., Zhang, X.M., Cao, W.J. and Sheng, X.Y. (2013) Correlation Analysis between Post-Stroke Constipation and Brain Injury. Journal of South Medical University, 33, 117-120.

[9] Su, Y.J., Zhang, X.Y., Zeng, J.S., Pei, Z., Cheung, R.T.F., Zhou, Q.P., Ling, L., Yu, J., Tan, J.L. and Zhang, Z.L. (2009) New-Onset Constipation at Acute Stage after First Stroke: Incidence, Risk Factors, and Impact on the Stroke Outcome. Stroke, 40, 1304-1309. http://dx.doi.org/10.1161/STROKEAHA.108.534776

[10] Jura, E. (1987) Gastrointestinal Disturbances in Stroke. Acta Neurologica Scandinavica, 76, 168-171. http://dx.doi.org/10.1111/j.1600-0404.1987.tb03562.x

[11] Johanson, J.F., Sonnenberg, A., Koch, T.R., et al. (1992) Association of Constipation with Neurologic Diseases. Digestive Diseases and Sciences, 37, 179-186. http://dx.doi.org/10.1007/BF01308169

[12] Bracci, F., Badiali, D., Pezzotti, P., Scivoletto, G., Fuoco, U., Di Lucente, L., Petrelli, A. and Corazziari, E. (2007) Chronic Constipation in Hemiplegia Patients. World Journal of Gastroenterology, 13, 3967-3972.

[13] Martin, R.E. and Sessle, B.J. (1993) The Role of the Cerebral Cortex in Swallowing. Dysphagia, 8, 195-202. http://dx.doi.org/10.1007/BF01354538

[14] Brandt, L.J., Schoenfeld, P., Prather, C.M., Quigley, E.M.M., Schiller, L.R. and Talley, N.J. (2005) Evidenced-Based Position Statement on the Management of Chronic Constipation in North America. American Journal of Gastroenterology, 100, S1-S21. http://dx.doi.org/10.1111/j.1572-0241.2005.50613_2.x 
Scientific Research Publishing (SCIRP) is one of the largest Open Access journal publishers. It is currently publishing more than 200 open access, online, peer-reviewed journals covering a wide range of academic disciplines. SCIRP serves the worldwide academic communities and contributes to the progress and application of science with its publication.

Other selected journals from SCIRP are listed as below. Submit your manuscript to us via either submit@scirp.org or Online Submission Portal.
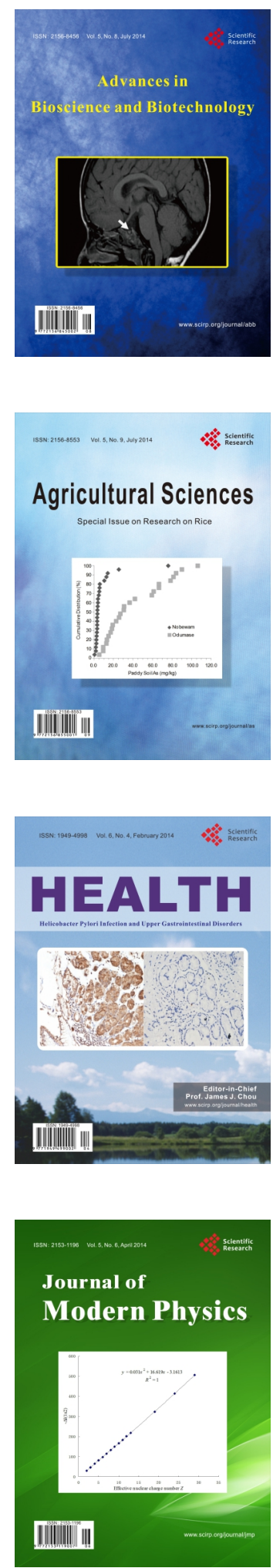
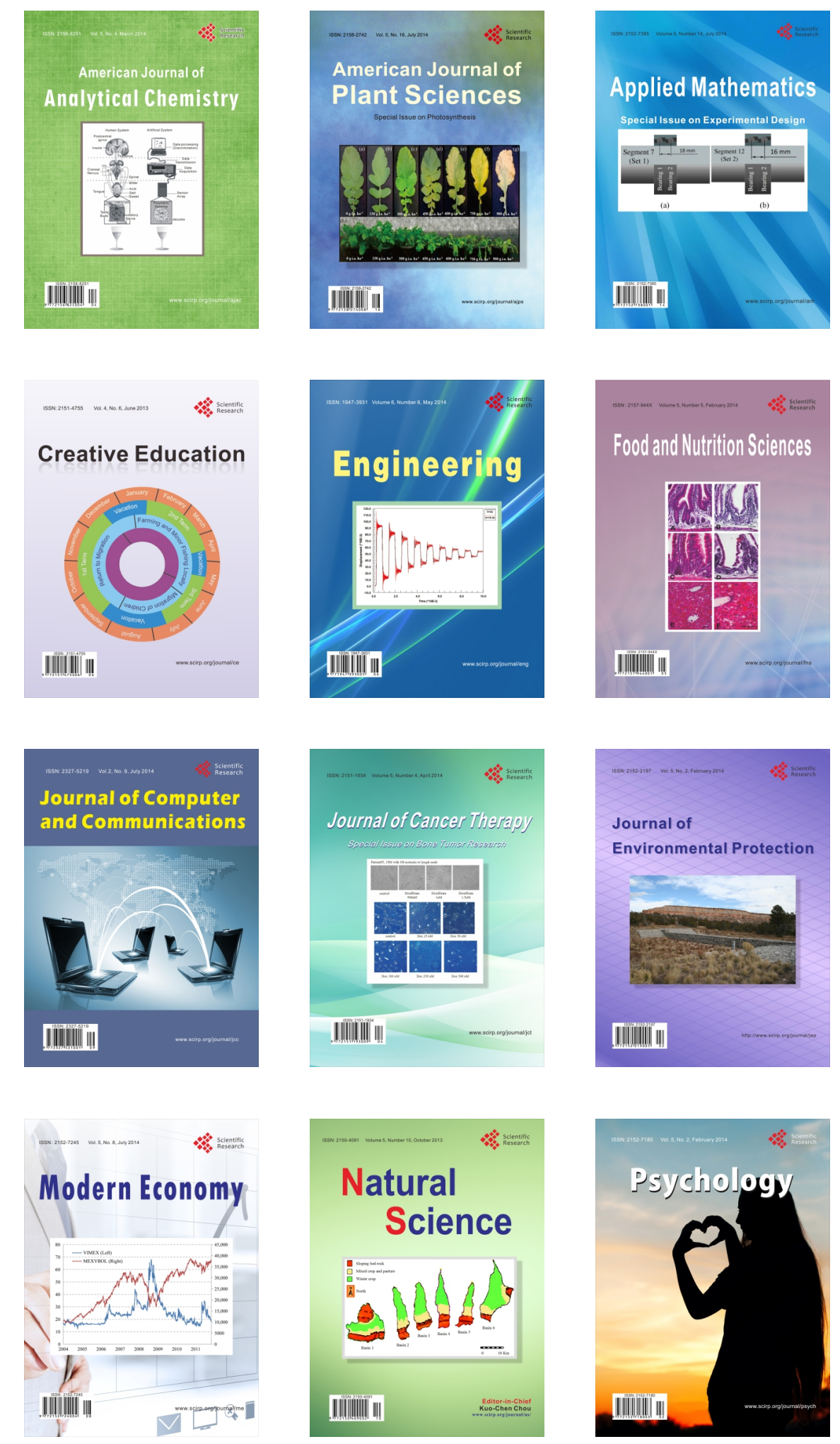